\title{
Inhibition of electromyographic activity in human triceps surae muscles during sinusoidal rotation of the foot
}

\author{
WILLIAM FREEDMAN ${ }^{1}$ AND RICHARD HERMAN \\ From the Department of Rehabilitation Medicine, Temple University School of Medicine, \\ and Krusen Research Center, Moss Rehabilitation Hospital, Philadelphia, Pennsylvania, U.S.A.
}

SYNOPSIS Torque and electromyographic (EMG) responses to sinusoidal rotations of the foot were measured. The frequency range of the movements was $0.5 \mathrm{~Hz}$ to $15 \mathrm{~Hz}$ at amplitudes ranging $\underset{\sigma}{\omega}$ between 1 and 10 degrees. At frequencies above $7 \mathrm{~Hz}$, the EMG activity did not follow individual foot rotation cycles. The EMG activity was inhibited whenever the peak torque was large with respect to the first cycle peak torque. Dantrolene sodium reduced the torque developed in the $\mathscr{\infty}_{\infty}^{\omega}$ triceps surae, allowing the EMG activity to follow individual stretch cycles. As the drug was metabol- $\rightarrow$ ized, the EMG activity returned to the character seen in the pre-drug control-that is, inhibition on $\mathscr{W}_{\sigma}$ alternate stretch cycles. It is concluded that the EMG inhibition phenomenon can be attributed in 0 part to force receptors in muscle but that these receptors are not the sole contributors to the inhibition.

Excitability of the spinal motoneurone pool in animals has been extensively studied using one of the following basic methods: (1) myography and electromyography, (2) monosynaptic testing with conditioning and test stimuli, and (3) intracellular recording techniques (Granit, 1970). These methods have provided tools by which investigators have attempted to delineate the spinal cord response to a known afferent input. However, the afferent signals generally have been set up by electrical stimuli which, although easily controlled, do not represent functional or physiological information.

Investigations of human spinal cord excitability have concentrated on the use of Hoffman and tendon reflexes (Magladery and Teasdall, 1958; Diamantopoulos and Olsen, 1965; Herman, 1969, and others). These 'unnatural' stimuli present the same problems of interpretation as those discussed by P. B. C. Matthews in the context of animal experimentation-that is, that 'the spinal centres appear to be duly organized

1 Address for correspondence: William Freedman, Krusen Research Center, Moss Rehabilitation Hospital, 12th Street and Tabor Road, Philadelphia, Pennsylvania 19141, U.S.A. (Accepted 3 September 1974.) so that they can only emit "sensible answers even though the mixture of afferent inputs elicited by electrical stimulation presents them with a "stupid question"' (Matthews, 1972).

For the above reason, the experiments described here were designed to study the response of the human motoneurone pool to repetitive stretch stimuli. Sinusoidal rotation of the foot of various amplitudes and frequencies was used as the stimulus to elicit a response measured as electromyographic (EMG) activity of the ankle musculature and as developed ankle torque. The particular phenomenon to be described is similar to observations made in gastrocnemius motoneurones of anaesthetized cats whose muscles were stretched repetitively (range $2-6 \mathrm{~mm}$ ) with triangular waveform displacements (Homma et al., 1970). Intracellular recordings indicated that the impaled motoneurones discharged once during each triangular displacement until the $\sigma$ stretching frequency approached $10 \mathrm{~Hz}$. Above $\mathrm{N}$ this frequency, membrane depolarization was frequency limited in that it did not occur on every cycle of muscle stretch.

The occurrence of frequency limitation of 
EMG activity in the triceps surae muscles of man has been previously noted to occur during high frequency $(7-12 \mathrm{~Hz})$, large amplitude (10 degrees) foot rotations (Herman et al., 1973). However, the effect of this phenomenon on developed ankle torque could only be inferred due to the presence of large inertial torques inherent in the experimental equipment. Moreover, the origin of the inhibition of EMG activity was not investigated. This investigation on human subjects describes the effect of widespread inhibition of the motoneurone pool on ankle torque and the probable cause of the frequency-limiting behaviour observed in EMG activity.

\section{METHODS}

SUBJECTS AND PREPARATION Five healthy adults, two patients with spinal cord lesions, and eight patients with hemiplegia took part in these experiments. Patients were selected for the study if they exhibited hyperreflexia without other major diagnosed disorders such as cardiac involvement. Normal subjects with active tendon jerks were selected. The subject's left lower limb (or the paretic lower limb in hemiplegics) was fastened by straps and a sandal to a servo-controlled positioning device while the subject lay prone (or supine where the prone position was uncomfortable). The rotational axis of the positioning device was aligned $1 \mathrm{~cm}$ below and $1 \mathrm{~cm}$ anterior to the lateral malleolus as a standard approximation to the ankle axis. Surface electrodes were placed over the lateral portion of the soleus (Sol), medial gastrocnemius (MG), and tibialis anterior (TA) muscles. The electrodes were placed with each pair parallel to the direction of the muscle fibres with interelectrode distance about $3 \mathrm{~cm}$.

EQUIPMENT The foot rotational device was a hydraulically powered position servomechanism which, for this protocol, moved sinusoidally through a constant amplitude ranging from $1^{\circ}$ to $10^{\circ}$ with a frequency range of $0.5 \mathrm{~Hz}$ to $15 \mathrm{~Hz}$. The high frequency constraint $\left(15 \mathrm{~Hz}\right.$ at $10^{\circ}$ amplitude) was a function of the mass of the mechanical system and of the subject's foot. A diagram of the device is shown in Fig. 1.

The positioning device provided a measure of foot position and of torque generated by the ankle musculature. Torque was measured by a strain gauge reaction torque sensor (Lebow Associates; Troy, Michigan), while position was monitored by means of a potentiometer. Surface EMG electrode signals (Beckman, Anaheim, Cal.) were conditioned using amplifiers (Teca, Model N8; New York) set at a gain of 3000 with a pass band of $5 \mathrm{~Hz}-1600 \mathrm{~Hz}$. The three EMG signals, ankle torque, and foot position were monitored on an ultra-violet recorder (Honeywell Visicorder Model 2106; Denver, Col.).

ANALYSIS OF TORQUE DATA The torque sensing device measured the total torque acting about the ankle axis. This includes the torque due to rotating the mass of the foot positioning device and the subject's foot-that is, inertial torque-as well as the active and passive torques due to the subject's ankle musculature. Since only the muscle-produced torque was of interest, a method for eliminating inertial torque was developed. A measure of inertia independent of the strain gauge torque sensor was used

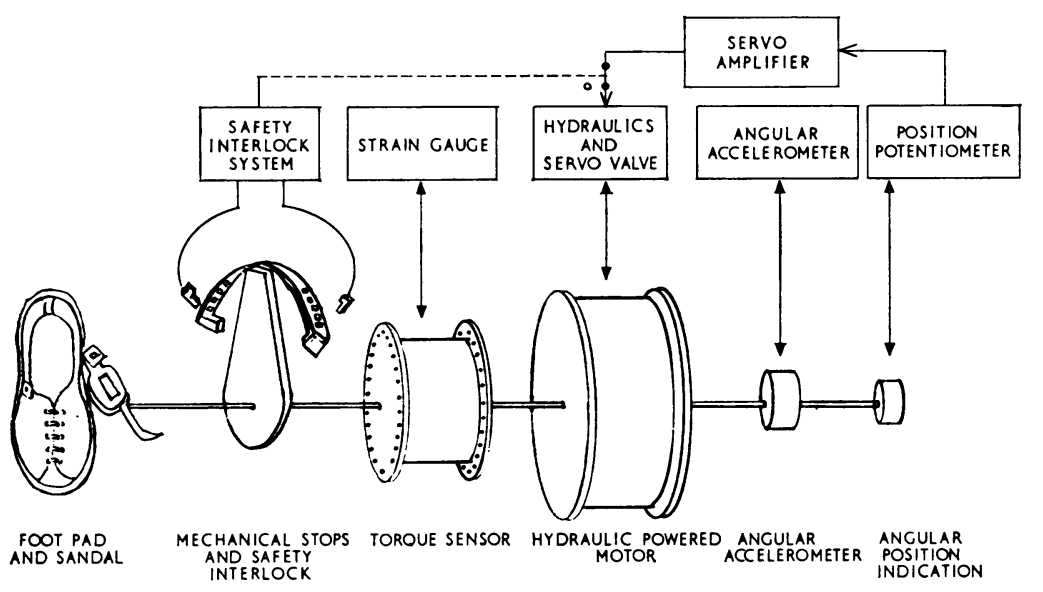

FIG. 1 Schematic diagram of the hydraulic foot positioning device and its servocontrol. 
for this purpose. An angular accelerometer (Schaevitz Engineering; Camden, N.J.) mounted on the oscillating member of the foot positioning device (Fig. 1) produced a voltage output $V_{a}=k \alpha$, where $k$ is the scale factor (volts $\left./ \mathrm{rad} / \mathrm{s}^{2}\right)$ and $\alpha$ is the acceleration $\left(\mathrm{rad} / \mathrm{s}^{2}\right)$.

The position of the sandal on the foot positioning device varied from subject to subject as did the foot mass of each individual. These variations had to be considered since they produced appreciable changes in the torque sensed by the strain gauge. Thus, the foot weight of each subject was estimated using water displacement to determine foot volume, as well as the density of the foot as determined by Contini (1970). From calibration curves, the accelerometer output voltage required to adjust for the subject's ankle axis and foot weight could then be determined. The strain gauge torque sensor output may be expressed as

$$
V_{s g}=k_{1}\left(T_{m}+T_{i}\right)
$$

where $k_{1}$ is the unit scale factor $(\mathrm{V} /$ Newtonmetre)

$T_{m}$ is the torque due to the ankle musculature $(\mathrm{Nm})$

$T_{i}$ is the torque due to the system and foot inertia $(\mathrm{Nm})$

The accelerometer output was adjusted to be $V_{a}=k_{1} T_{i}$ before the subject's foot was inserted into the sandal. With the subject's foot fastened to the positioning device, the corrected torque output voltage could be written as follows:

or

$$
V_{s g}-V_{a}=k_{1}\left(T_{m}+T_{i}\right)-k_{1} T_{i}
$$

$$
V_{s g}-V_{a}=k_{1} T_{m}
$$

This is a measure of the torque developed by the ankle musculature. Without this torque correction, ankle torque could be determined up to $3 \mathrm{~Hz}$ before the effect of inertia became dominant. With this method, ankle torque was recorded up to $15 \mathrm{~Hz}\left(10^{\circ}\right.$ amplitude of oscillation) with some 'noise' on the torque record due to imperfect inertia compensation.

Nerve Blocks In order to isolate the agonist from antagonist effects at the ankle joint, a nerve block was performed on two subjects. The ankle extensor muscle group was isolated by chemically denervating the common peroneal nerve at the head of the fibula (using $2 \%$ xylocaine). The nerve was located and identified using a constant current stimulator (Teca Chronaximeter; New York, New York). The location and perfusion of the nerve were accomplished with a hypodermic needle electrode.
DRUG STUDY Dantrolene sodium has been shown to reduce the force generated in skeletal muscle by interfering with the excitation-contraction coupling reaction in extrafusal muscle fibres (Putney and Bianchi, 1973). Six subjects including two nonpatients were used for this portion of the study. The protocol was carried out on these subjects as a control. Then a loading dose $(2 \mathrm{mg} / \mathrm{kg})$ of the drug was administered perorally. Tendon jerk responses were used to monitor the drug effect. After the tendon jerk amplitude had been reduced by $75 \%$ (1.5-2 hours after drug ingestion), the protocol was again performed. In one instance, a second post-drug test was performed about 2.5 hours after the drug followed (five hours after the drug) by another test to show the return to pre-drug torque values.

HUMAN SUBJECT SAFETY The hydraulically powered device used to position the subject's foot was designed with several electrical and mechanical interlock features to ensure the subject's safety (Fig. 1). The last safety device, which was set within the range of motion of each subject's foot, was a pair of solid mechanical stops which, if not in place, opened the servomechanism control loop. Electrically, the leakage current from each subject lead, to each system earth (ground) was less than $10 \mu \mathrm{A}$.
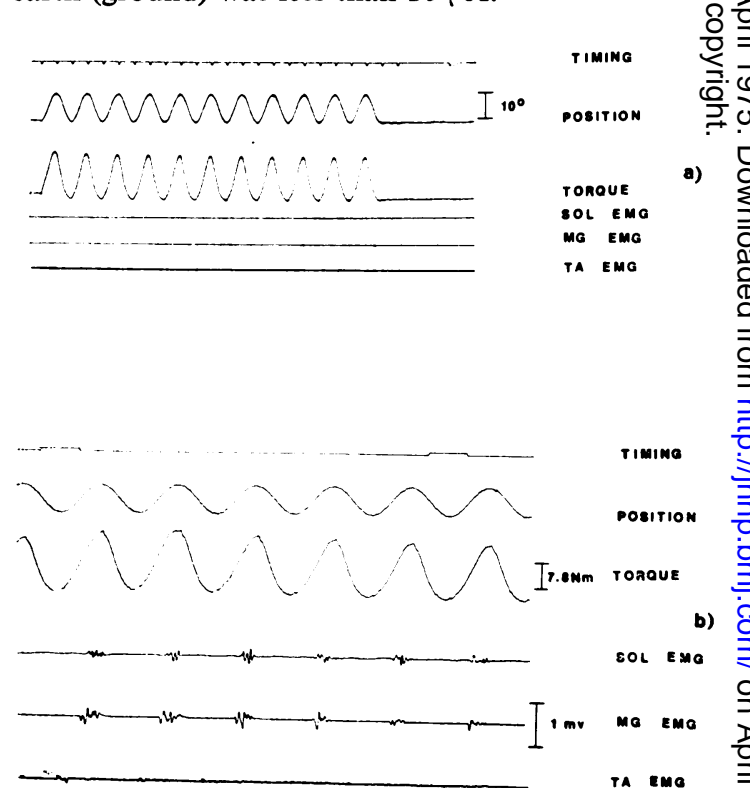

FIG. 2 Torque and EMG responses to sinusoidal foot rotation in a hemiparetic subject. In (a) and (b), the timing pulse interval indicates $1 \mathrm{~s}$. Note that at $0.5 \mathrm{~Hz}$ (a), no EMG activity is indicated; however, at $5 \mathrm{~Hz}$ (b), EMG activity occurs in the ankle extensor muscles during each stretch cycle. 
EXPERIMENTAL PROCEDURE The subject lay prone or supine with his ankle axis aligned with the rotational axis of the positioning device. His foot was set to a starting angle (usually $10^{\circ}$ plantar-flexion) which would then correspond to the most relaxed muscle length-for example, for an amplitude of foot rotation of $5^{\circ}$, the positioning device would start from $10^{\circ}$ plantar-flexion and oscillate between that position and $5^{\circ}$ plantar-flexion. The number of cycles of foot rotation was determined by presetting a counter (usually this was set at 10 cycles). The range of frequencies $(0.5-15 \mathrm{~Hz})$ was presented at a particular amplitude of foot rotation (range $1^{\circ}-10^{\circ}$ ). Instructions were given to the subject to relax and to assume a comfortable head and arm position before each group of foot oscillations. A fixed time interval (1.5 minutes) was allowed between stretch sequences to control muscle recovery time.

\section{RESULTS}

EMG RESPONSES TO HIGH FREQUENCY FOOT ROTATION The ankle flexor and extensor muscle EMG activity and the resulting ankle torque changed character in response to increasing the frequency of foot sinusoidal rotation at any one amplitude. For frequencies below $1 \mathrm{~Hz}$, throughout the range of amplitudes, the developed torque was due to rheological properties since no EMG activity was detectable (Fig. 2). At some frequency above $1 \mathrm{~Hz}$, depending upon the level of afferent support and the state of excitability of the subject's motoneurone pool, EMG activity could be observed during each stretch cycle. Two exceptions to this were noted: (1) many normal subjects demonstrated EMG activity only on the first cycle of repetitive ankle rotation; (2) some subjects with spinal cord lesions exhibited the large, spasmodic EMG activity associated with flexor withdrawal responses; however, in these subjects, chemical denervation of the flexor musculature (see below) resulted in phasic EMG responses to each cycle of repetitive foot movement.

In both normal and hemiplegic subjects, within the frequency range $1-5 \mathrm{~Hz}$, peak torque corresponding to the first stretch cycle was larger than the succeeding peaks (Lennerstrand and Thoden, 1968; Herman et al., 1973). The EMG corresponding to this torque had a stable time relationship to the causal position change-that is, the latency from the most plantar-flexed foot

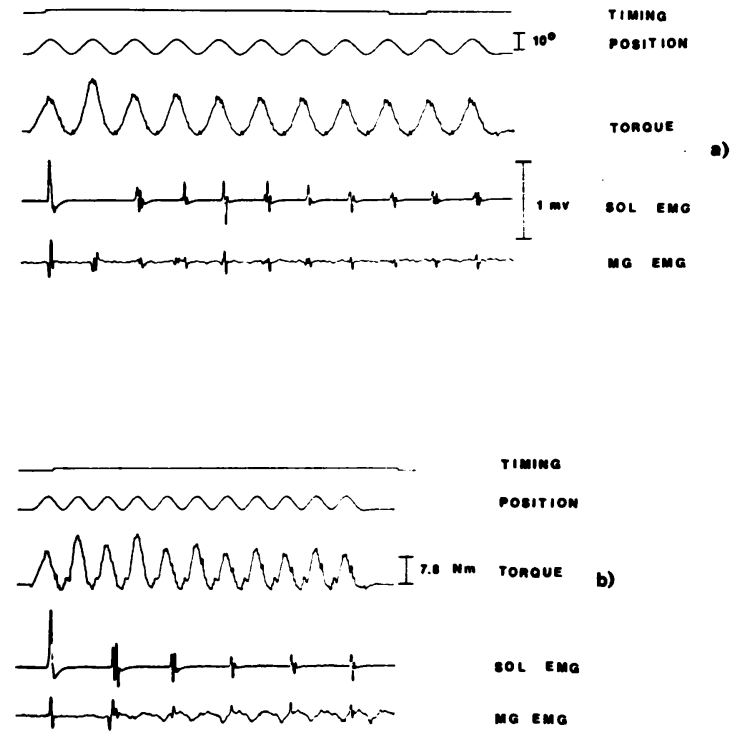

FIG. 3 Torque and EMG responses to high frequency foot rotation. Note that in (a) the second torque peak is higher than the other peaks and that the soleus EMG is inhibited during the second stretch cycle; however, the medial gastrocnemius EMG is not inhibited at this stretch rate $(9 \mathrm{~Hz})$. In (b), the torque peaks vary in amplitude in accordance with the EMG inhibition which occurs throughout the record. The medial gastrocnemius EMG is also inhibited on alternate stretch cycles; however, movement artefact is apparent at this frequency $(13 \mathrm{~Hz})$ after the fifth stretch cycle in the $M G$ record.

position to the peak of EMG activity was reasonably constant after the first stretch cycle. The first cycle EMG latency was always shorter than the latency on succeeding cycles.

As the foot rotation frequency was increased into the range of 7-9 Hz, an alternation of low and high peak torque values was observed. Concomitantly, the extensor (soleus and medial gastrocnemius) EMG activity was inhibited on alternate cycles-firing on those cycles during which low values of torque were obtained (Fig. 3a). The flexor muscle tibialis anterior was generally quiescent during the movements after an initial burst on the first stretch cycle. The alternate cycle inhibition of EMG activity lasted for several cycles; however, with higher frequencies, the effect would be observed for long 


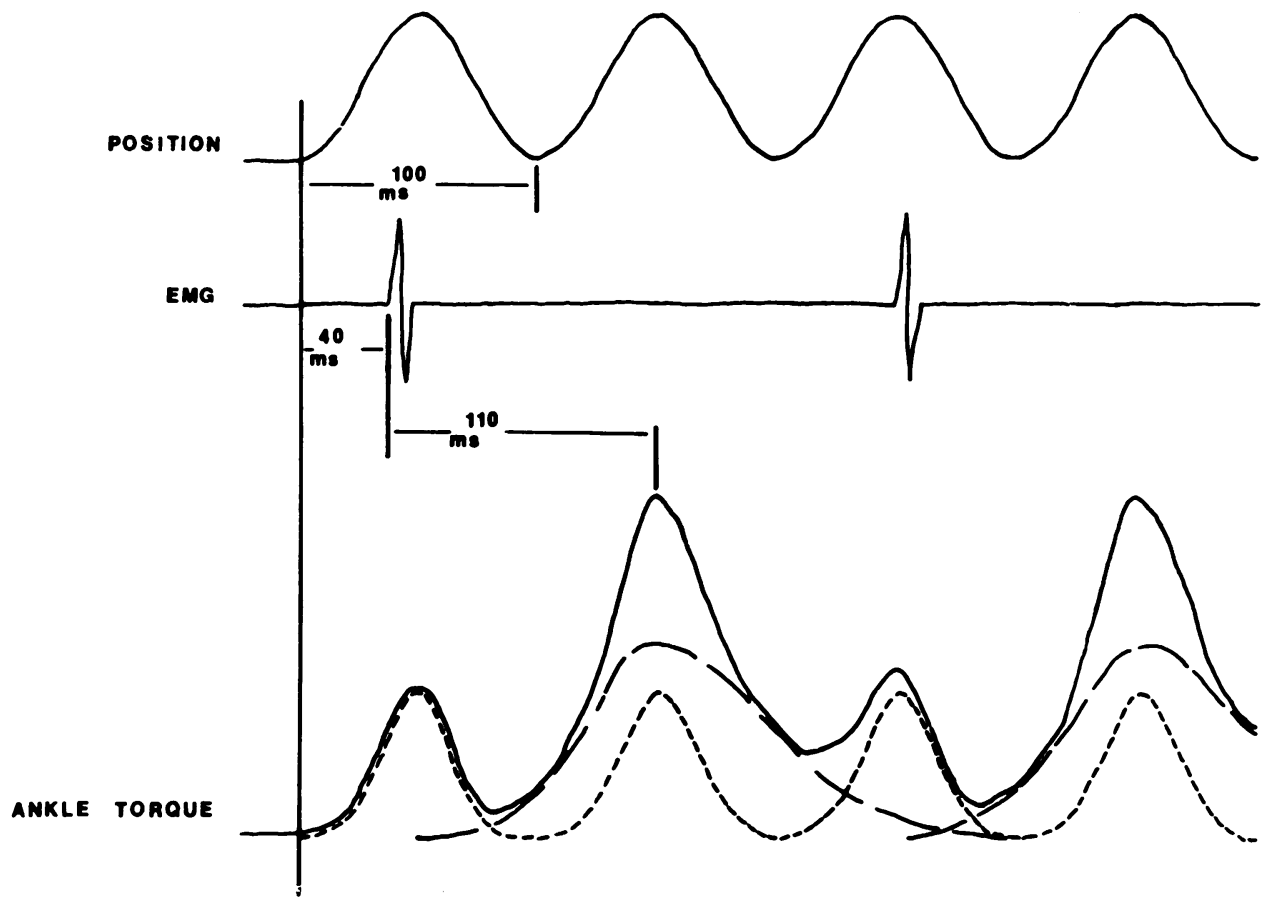

periods of time (Fig. 3b). Usually, the EMG activity more or less gradually assumed a steady firing pattern-that is, one burst per stretch cycle at a fixed latency with respect to the most relaxed length of the foot extensor muscles.

The repetitive foot movements were such that the $35-40 \mathrm{~ms}$ reflex loop transmission time for the human Achilles' tendon jerk was a substantial portion of the time for one stretch cycle. For example, at $10 \mathrm{~Hz}$ the time for one complete sinusoidal triceps surae stretch-relaxation cycle is $100 \mathrm{~ms}$. Moreover, the latency from the EMG burst to the peak of the torque generated in response to a large, transient muscle stretchfor example, an Achilles' tendon jerk-is approximately $110 \mathrm{~ms}$. Thus, it can be appreciated (Fig. 4) that at foot rotation frequencies of $10 \mathrm{~Hz}$, the effect of the EMG activity generated during the first stretching cycle may be exerted either as an eccentric or a concentric contraction on the second stretching cycle. This depends upon the latencies from (1) initiation of stretch to EMG activity (receptor set-up plus reflex loop time), and (2) EMG activity to peak of torque (contraction time). If the peak torque due to EMG activity occurs during stretch of the triceps surae muscles-that is, during movement toward foot dorsiflexion - the total ankle torque will be large on that cycle. If the peak of active torque occurs during muscle relaxation-that is, during movement toward foot plantar flexionthe total ankle torque on that cycle will be small (Joyce et al., 1969).

At rates of foot rotation near $12 \mathrm{~Hz}$, ankle $N$ torque generated by the EMG activity elicited N by the first stretch cycle affected the peak ankle 우 torque on the second and third stretching cycles. This is best illustrated in Fig. 5 where data for a 


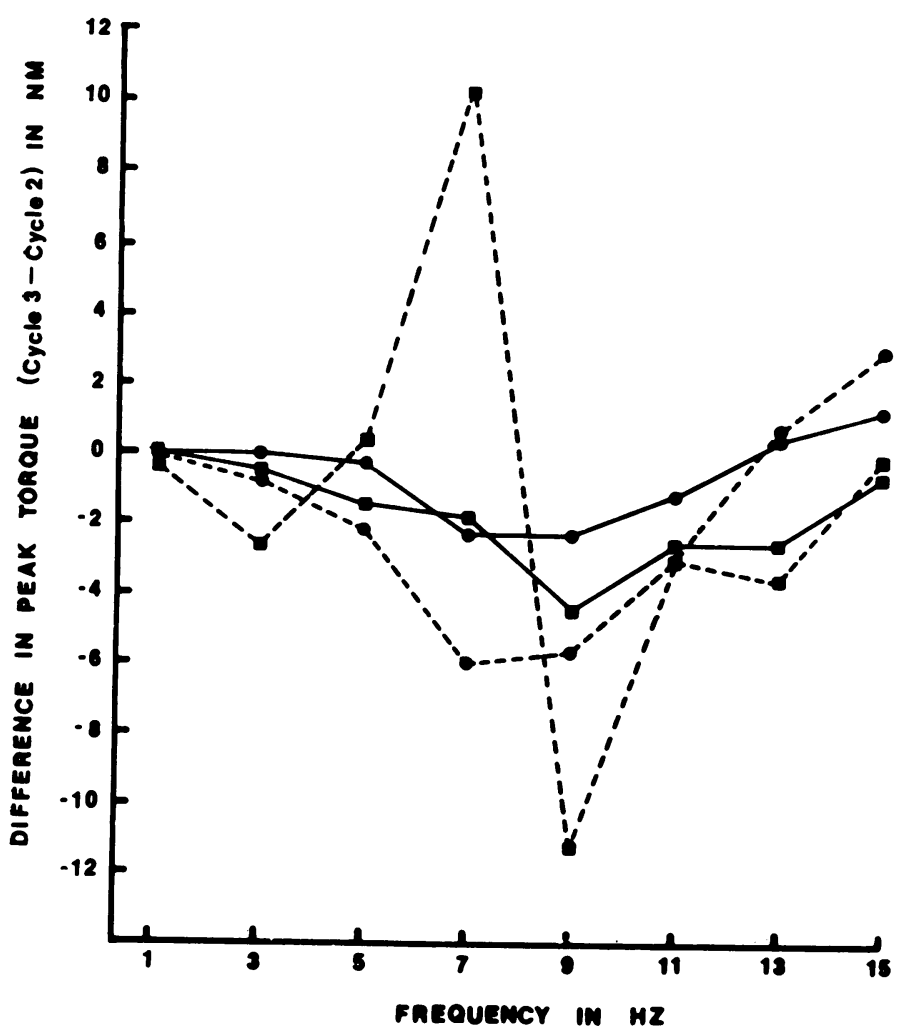

FIG. 5 Difference in peak torque values between third and second stretch cycles at various frequencies of stretch. Note that there is a minimum for each curve which falls in the range 7-9 Hz. This indicates that EMG activity which occurs during the first stretch cycle has an increasing effect on the third stretch cycle as the stretch frequency increases beyond $9 \mathrm{~Hz}$. Note that above $7 \mathrm{~Hz}$, EMG activity is inhibited on the second stretch cycle. - $5^{\circ}$. 10 $10^{\circ}$. Normal subject. --- Hemiplegic subject.

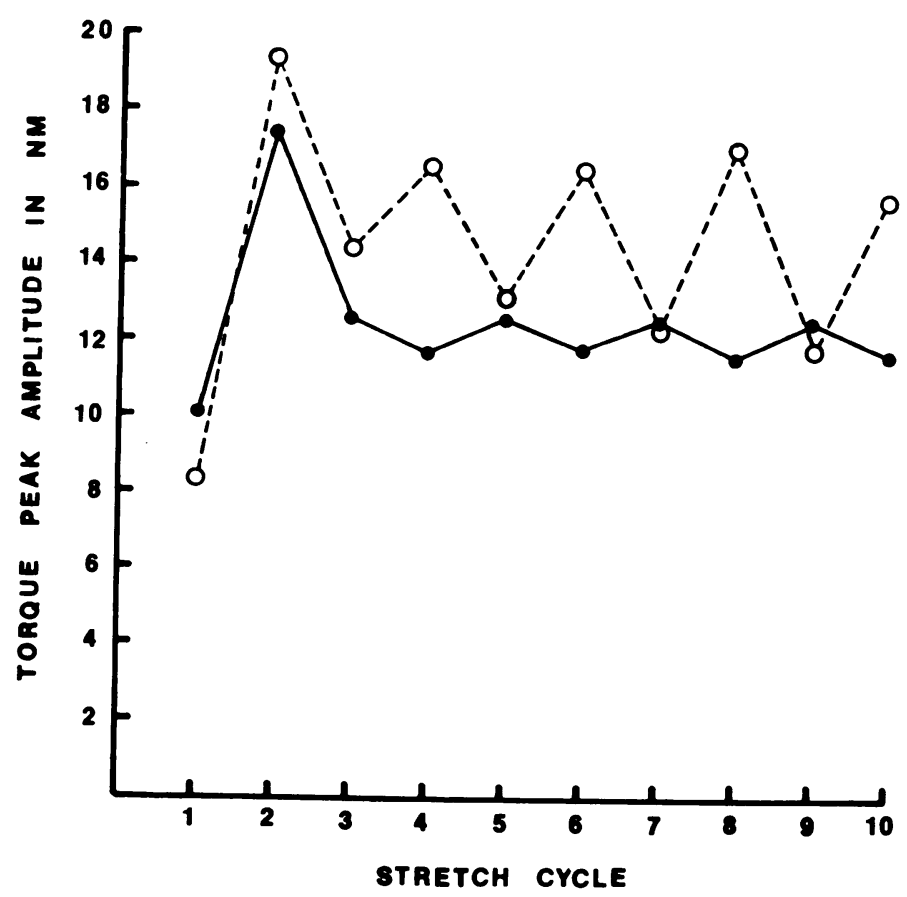

FIG. 6 Torque amplitude for each stretch cycle of $15 \mathrm{~Hz}, 10^{\circ}$ foot rotation in a quadriplegic subject. The control values indicate a stable peak torque value after the second stretch cycle. This was the result of minimal EMG activity. After a common peroneal $(C P)$ nerve block the torque peaks alternate between the control value and some higher level. The EMG activity after the nerve block is inhibited on alternate cycles resulting in the peak torque values which correspond to minimal EMG activity during the control test. Control. O CP block. 


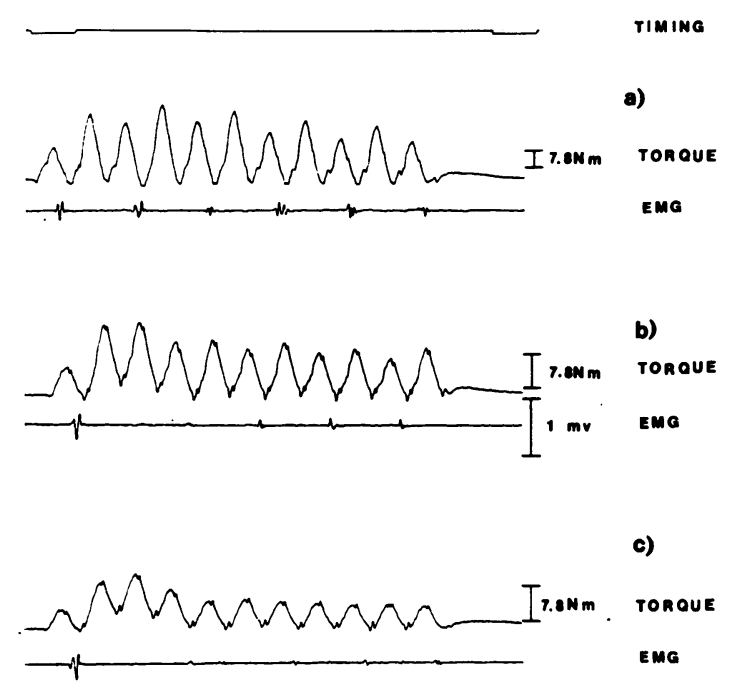

FIG. 7 Effect of amplitude of foot rotation on inhibition of EMG activity. (a), (b), and (c) show torque and soleus EMG data in response to $13 \mathrm{~Hz}$ sinusoidal foot rotations of $10^{\circ}, 5^{\circ}$, and $3^{\circ}$ amplitudes, respectively. Note the changing level of peak torque values whenever EMG activity is prominent. Also note that the torque values during the early stretch cycles do not return to the base line torque, as explained in Fig. 4.

normal and a hemiplegic subject are presented. The difference in peak ankle torque between the second and third stretch cycles is plotted against frequency for two different amplitudes of foot rotation. These curves show a distinct minimum at approximately $9 \mathrm{~Hz}$ after which the torque of the third stretch cycle clearly begins to dominate over the second cycle peak torque. This reflects the increasing effect of the first cycle EMG activity on the third cycle torque values as the frequency of foot oscillation was raised above $9 \mathrm{~Hz}$. When the third cycle peak torque clearly became dominant over the second cycle torque, EMG activity was inhibited on the second and third stretching cycles (see Fig. 8a).

In one of the patients with a spinal cord lesion, inhibition of EMG activity at high rates of foot movement was not observed. In fact, almost no EMG activity was seen. However, when the flexor muscles were chemically denervated (common peroneal nerve block with $2 \%$ xylocaine), the phenomenon was observed as seen by the alternations in peak ankle torque for
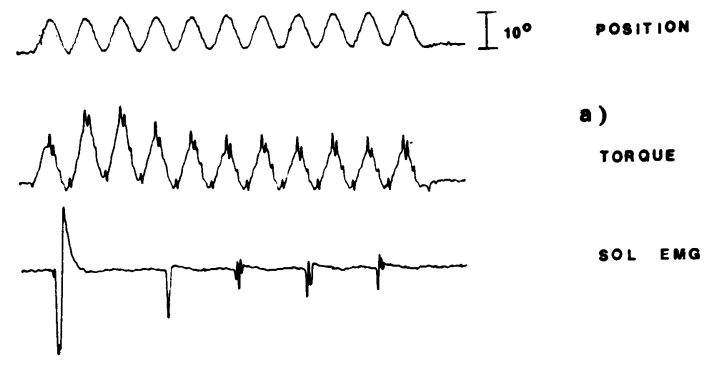

a)

TOR OUE
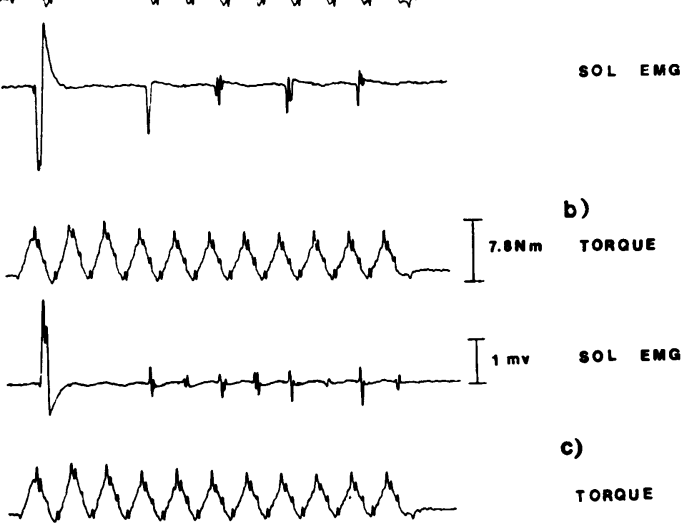

c)

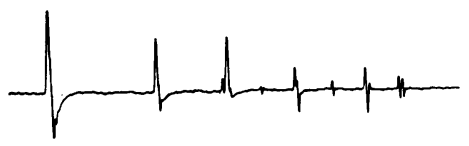

TORQUE

SOL EMG

FIG. 8 Effect of dantrolene sodium on torque an\$ EMG activity in response to $13 \mathrm{~Hz}$ sinusoidal foo rotation of $10^{\circ}$ amplitude. (a) Shows pre-drug controb records, while the data of parts (b) and (c) were re corded 2.5 hours and five hours after drug ingestion, respectively. Note that the jagged appearance of the torque records is due to a combination of recording system noise and of imperfect inertia compensation.

each cycle of foot rotation (Fig. 6). Note that the low torque values occurring on alternate stretch cycles during the common peroneal nerve block test correspond to the values measured during the control. This is expected, since little EMG activity was recorded during the control test and, during the common peroneal nerve block test, EMG activity was inhibited on alternate stretch cycles.

EFFECT OF AMPLITUDE OF FOOT ROTATION ON INHIBITION OF EMG ACTIVITY When the ampli- o tude of foot rotation was reduced below $10^{\circ}$, the torque induced by muscle stretch was also reduced. This had been shown previously (Herman et al., 1973) to occur in a non-linear fashionthat is, at any given frequency of foot oscilla- 
tion, a decrease in the amplitude of rotation produced a non-proportional change in ankle torque. Although alternate cycle inhibition of EMG activity may have been present at a particular amplitude - for example, $10^{\circ}$ at $13 \mathrm{~Hz}-$ the magnitude of foot rotation could be reduced to a level which caused the EMG firing to disappear entirely after the first cycle (Fig. 7).

EFFECT OF ADMINISTRATION OF DANTROLENE soDIUM The drug dantrolene sodium affects the efficiency of muscle by interfering with the excitation-contraction coupling reaction of striated skeletal muscle. This means that the force developed by a muscle in response to an electrical or mechanical stimulus will be less after drug ingestion than the force developed in a pre-drug control (Monster et al., 1973).

Before the administration of dantrolene sodium, data derived from one normal subject and three hemiplegic patients exhibited inhibition of EMG activity during foot oscillations greater than $7 \mathrm{~Hz}$ (Fig. 8a). After the drug was administered, similar experimental protocol resulted in decreased torque levels and modified EMG behaviour. The EMG activity was not inhibited to the extent it had been in the predrug control experiments. Bursts of EMG signals tended to 'fill-in' those cycles during which inhibition had previously occurred (Fig. 8b). Five hours after drug ingestion, the EMG inhibition was tending to return to its pre-drug character (Fig. 8c). Note, however, that the drug had not been fully metabolized since the torque levels were still low with respect to the control values.

\section{DISCUSSION}

The main finding described here is the inhibition of electromyographic activity during foot rotation at frequencies above $7 \mathrm{~Hz}$. The result of this failure of the reflex loop to follow individual sinusoidal stretches of muscle is observed in the alternating levels of peak torque developed by the ankle musculature. Similar frequencylimiting behaviour was observed during intracellular recordings of gastrocnemius motoneurones in anaesthetized cats (Homma et al., 1970). These authors indicated a failure of motoneurones to follow individual triangular muscle stretches at frequencies above $10 \mathrm{~Hz}$. They attributed the inhibition to a progressive decrease in groups Ia, Ib, and II receptor discharges, as stretch frequency was increased with relatively less of a decrease in groups Ib and II than in group Ia. They also discussed probable supraspinal effects involved in the motoneurone discharge frequency-limiting phenomenon.

The EMG inhibition noted in the human ankle myotatic reflex loop was tentatively attributed to the action of force sensitive receptors. With this hypothesis, experiments were performed in which ankle torque was reduced by (1) reduction of foot rotation angle - that is, a reduced input to the system-and by (2) administration of a drug which reduces ankle torque with respect to control values although the external stimulus to the reflex system is the same both before and after drug ingestion.

Reducing the foot rotation angle does cause a decline in ankle torque (Fig. 7); however, the causal mechanism cannot be inferred from this data because several afferent signal generators would be expected to change discharge rate as a function of input amplitude. The mathematical nature of variations in the amplitude of a sinusoidal position signal ensures changes as well in the velocity and acceleration of foot rotation. This suggests that receptors sensitive to position (muscle spindles and joint receptors), to velocity (muscle spindles and joint receptors), to force (Golgi tendon organs), and perhaps to acceleration (muscle spindles) would all be affected by a change in amplitude of the repetitive foot movements. The experiments in which input amplitude was changed reflected these receptor output variations in that the occurrence of EMG inhibition was not proportional to the foot rotation amplitude. In fact, the alternate cycle EMG inhibition observed during $10^{\circ}$ foot movements did not tend to decrease in occurrence at $5^{\circ}$ foot oscillations. At a given frequency, if the amplitude of movement was enough to generate EMG activity on more than the first stretch cycle, the EMG inhibition phenomenon occurred.

The changes in foot rotation amplitude resulted in concomitant changes in the amplitude of ankle torque. Reduction in torque level would decrease force receptor discharge, which, in turn, would tend to increase the excitability level of the motoneurone pool. Thus, if the EMG 
inhibition phenomenon were a function only of peak torque, reduction of the input amplitude would tend to increase the occurrence of EMG on each stretch cycle. Since this tendency toward EMG activity on each stretch cycle did not occur, it is suggested that peripheral mechanisms in addition to force receptors must also be considered as contributing to the level of excitability of the motoneurone pool.

Use of the peripherally acting muscular relaxant dantrolene sodium (Herman et al., 1972; Monster et al., 1973; Putney and Bianchi, 1973) provided a reduction in developed ankle torque without changing the input to the reflex system (Fig. 8). It appears from these data that the EMG inhibition is a function of the torque amplitude. This implicates force sensitive receptors as at least partially responsible for the phenomenon.

Supraspinal influences in the production of alternate cycle EMG inhibition seem to be minimal. This was shown in the quadriplegic patient who had been diagnosed as having a (physiologically) complete spinal cord lesion. After the inhibitory effect of the antagonist tibialis anterior was removed by chemical denervation of the common peroneal nerve, the EMG activity of the extensor muscles could not follow individual stretch cycles when the input foot oscillations became greater than $7 \mathrm{~Hz}$.

Because of constraints imposed by the experimental protocol, only indirect evidence can be cited for specific receptor function. It is clear, however, that the antagonist muscle is not the cause for the EMG inhibition observed during repetitive foot oscillations above $7 \mathrm{~Hz}$. This was shown by chemical denervation of the antagonist muscle, after which the agonist muscle EMG inhibition was, if anything, more pronounced.

It is also clear that the observed EMG inhibition is dependent on force sensitive receptors in muscle; however, the relationship of force receptors to the inhibition phenomenon is not independent of other afferent signals. This is suggested by the fact that the level of peak torque at which EMG inhibition occurs with a $10^{\circ}$ foot rotation is about one half the peak torque value for which the inhibition occurs with a $5^{\circ}$ foot rotation (Fig. 7). Note that this is so for both total torque and active torque (see Fig. 4). Thus, the EMG inhibition which occurs in the human lower extremity at foot rotation frequencies greater than $7 \mathrm{~Hz}$ is a function of the activation of force sensitive receptors in muscle continuously modulating the spinal milieu set up by other peripheral afferent information (Jansen and Rudjord, 1964; Houk and Henneman, 1967). This is the working hypothesis for understanding motoneurone excitability in response to repetitive stretch stimuli to which these experiments have led.

The authors would like to thank Mr Paul Macks for the audio-visual work and Mrs Eileen Klaus for her fine cooperation in preparing this report. The work has been supported by grant no. 16P56804/3-09 from the Department of Health, Education, and Welfare, the Social and Rehabilitation Service, Washington, D.C.

\section{REFERENCES}

Contini, R. (1970). Body Segment Parameters (Pathological). Final Report. Technical Report No. 1584.03. New York University, School of Engineering and Science: Bronx.

Diamantopoulos, G. E., and Olsen, P. Z. (1965). Motoneurone excitability in normal subjects and patients with abnormal muscle tone. Proceedings of the Sixth International Congress of Electroencephalography and Clinicaß Neurophysiology, Vienna, 1965. American Elsevier: New York.

Granit, R. (1970). Basis of Motor Control, pp. 100-103규 जे Academic Press: London.

Herman, R. (1969). Relationship between the $\mathrm{H}$ reflex and the tendon jerk response. Electromyography (Louvain), 4, 359-370.

Herman, R., Mayer, N., and Mecomber, S. A. (1972). Clinical pharmaco-physiology of dantrolene sodium. American Journal of Physical Medicine, 51, 296-311.

Herman, R., Freedman, W., Monster, A. W., and Tamai, Y. (1973). A systematic analysis of myotatic reflex activity in human spastic muscle. In New Developments in Electromyography and Clinical Neurophysiology, vol. 3, pp. 556578. Edited by J. E. Desmedt. Karger: Basel.

Homma, S., Ishikawa, K., and Stuart, D. G. (1970). Motoneurone responses to linearly rising muscle stretch. American Journal of Physical Medicine, 49, 290-306.

Houk, J., and Henneman, E. (1967). Responses of Golgi tendon organs to active contractions of the soleus muscle of the cat. Journal of Neurophysiology, 30, 466-481.

Jansen, J. K. S., and Rudjord, T. (1964). On the silent period and Golgi tendon organs of the soleus muscle of the cat. Acta Physiologica Scandinavica, 62, 364-379.

Joyce, G. C., Rack, P. M. H., and Westbury, D. R. (1969). The mechanical properties of cat soleus muscle during controlled lengthening and shortening movements. Journal of Physiology, 204, 461-474.

Lennerstrand, G., and Thoden, U. (1968). Dynamic analysis of muscle spindle endings in the cat using length changes of different length-time relations. Acta Physiologica Scandinavica, 73, 234-250. 
Magladery, J. W., and Teasdall, R. D. (1958). Stretch reflexes in patients with spinal cord lesions. Bulletin of the Johns Hopkins Hospital, 103, 236-241.

Matthews, P. B. C. (1972). Mammalian Muscle Receptors and their Central Actions, p. 333. Monographs of the Physiological Society No. 23. Arnold: London.

Monster, A. W., Herman, R., Meeks, S., and McHenry, J.
(1973). Cooperative study for assessing the effects of a pharmacological agent on spasticity. American Journal of Physical Medicine, 52, 163-188.

Putney, J. W., Jr, and Bianchi, C. P. (1973). Effect of dantrolene on E-C coupling in skeletal muscle. (Abstract.) Federation Proceedings, 32, 772. 